\title{
Cryopreservation of a soil microbiome using a Stirling Cycle approach - a genomic assessment
}

\section{Author names}

Giovanni Cafa (https://orcid.org/0000-0003-0819-2126) ${ }^{1}$, Juan Miguel Bonnin (https://orcid.org/0000-0002-5095-6719) 1 , Nicola Holden (https://orcid.org/0000-00027904-4529) ${ }^{2}$, Jacob G Malone ${ }^{3,4}$ (https://orcid.org/0000-0003-1959-6820), Tim H Mauchline (https://orcid.org/0000-0002-8590-2614) ${ }^{5}$, Ian Clark (https://orcid.org/00000002-1589-5420) ${ }^{5}$, Rodrigo Taketani ${ }^{5}$, Matthew J Ryan (https://orcid.org/0000-0002$3689-6609)^{1 *}$

\section{Affiliation}

${ }^{1} \mathrm{CABI}$, Egham, TW20 9TY

${ }^{2}$ SRUC, Department of Rural Land Use, Craibstone Estate, Aberdeen, AB21 9YA

${ }^{3}$ Molecular Microbiology, John Innes Centre, Norwich Research Park, Norwich, NR4 7UH

${ }^{4}$ University of East Anglia, Norwich, NR4 7TJ

${ }^{5}$ Sustainable Agriculture Sciences, Rothamsted Research, Harpenden, AL5 2JQ

\section{Corresponding author}

* Matthew J Ryan m.ryan@cabi.org

\begin{abstract}
Soil microbiomes are dynamic systems that respond to biotic and abiotic environmental factors such as those presented at seasonal scales or due to long-term anthropogenic regime shifts. These can affect the composition and function of microbiomes. Investigation of microbiomes can uncover hidden microbial roles in health and disease and discover microbiome-based interventions. Collections of soil samples are kept by various institutions in either a refrigerated or occasionally frozen state, but conditions are not optimised to ensure the integrity of soil microbiome. In this manuscript, we describe cryopreservation with a controlled rate cooler and estimate the genomic content of an exemplar soil sample before and after cryopreservation. The first hypothesis was to test the genomic integrity of the microbiome. We also enriched the soil sample with a liquid medium to estimate the growth of bacteria and compared their growth before and after cryopreservation. Sequence-based rRNA metabarcoding was used to demonstrate that the controlled rate cooler maintains intact the DNA content of the microbiome. Two methods of cryopreservation were applied and compared with control aliquots of soil. An optimised cryopreservation of soil samples is
\end{abstract}


essential for the development of microbiome research in order to retain stable, functionally intact microbiomes. Our results showed that metabarcoding of $16 \mathrm{~S}$ and ITS rRNA were useful methods to estimate successful cryopreservation. The soil microbiome after enrichment with liquid medium exhibited a similar response of cryopreserved soil and this was estimated with the comparison of the ten most abundant bacterial taxa. These findings support a successful process of cryopreservation and are promising for future use of this technology. To the best of our knowledge, this study is the first report of cryopreservation of soil using a Stirling cycle cooling approach.

\section{Introduction}

Soil microbiomes are dynamic systems that respond to biotic and abiotic environmental factors in a temporally and spatially dependent manner, such as those presented at seasonal scales or due to long-term anthropogenic regime shifts, affecting their composition and function and their environment $(1,2)$. They offer great potential to contribute to the sustainable intensification of agriculture and their application could reduce our dependence on the use of toxic chemicals in agriculture and stimulate a more sustainable application of environmental resources $(3,4)$. Microbiome research and application may impact anthropogenic-driven climate change (5) and developing such an understanding is essential to tackle challenges facing human society today, such as the management of natural ecosystems and the mitigation of climate change (6).

To ensure robust application of whole beneficial microbiomes, methods for their preservation are required. Ideally, such methods will include the preservation of biotic components, abiotic factors as well as the physical environment where organisms function, such as the space where chemical reactions occur. Altering these factors will likely influence the integrity and function of preserved microbiomes when are subsequently resuscitated. For example, the removal of keystone organisms encoding functional genes due to the use of a sub-optimal storage methodology could irreversibly and negatively affect biological networks if these genes encode critical functions in a given microbiome system (7). As such, preservation of the community complex is critical (8). Storage capacity is a significant challenge to this approach. It is not practical to store large amounts of material such as soil, hence an understanding of the amount of sample required to be representative of the microbiome is required. For example, in agriculture a single field encompasses multiples of localized microbiomes (9).

The development of preservation methods to conserve samples as representative 'snap shots' in time is becoming a crucial part of microbiome research, as the requirement for reference material to repeat and validate research outcomes and its utilisation as a source of material for potential commercial use increases (7). Proving that the functionality of the microbiomes remain intact after cryopreservation is therefore critical. There is evidence that only a fraction of the microbiome can survive traditional cryopreservation and many organisms must therefore be isolated and cultured under specific conditions before freezing. As such, there is a need to improve microbiome cryopreservation methods. 
The rapid advancement of nucleic acid sequencing technologies has precipitated an urgent assessment of the role of biobanks to preserve microbiomes and underpin research to unlock their functional potential (7). Preservation of metagenomes from these samples allows microbiomes to be described in unprecedented detail and the functional potential of a given microbiome to be deciphered. However, the analysis of nucleic acids does not provide information on the viability of organisms, even though RNA analysis does give insights into microbial activity at the time of preservation.

When considering microbiome preservation there are two questions that need to be answered: (i) what should be preserved, and (ii) what is the best way of preserving it? (7). Historically, soil samples have been frozen and stored in mechanical freezers without any control of the rate of freezing. The application of a sub-optimal approach can compromise the microbial communities present as some will not survive the freezing process. The use of a Stirling cycle freezer for cryopreservation is considered to have significant advantages over traditional methodologies including nitrogen free operation, application of low cooling rates, reduction of sample contamination risks and control of ice nucleation (10). For the majority of organisms, applications of controlled cooling techniques reduce the prospect of ice damage that would otherwise compromise membrane integrity and cell viability. Assessment of cryopreservation regimes have included several tests to analyse viability of microorganisms in addition to the relative stability of genomic profiles. Stirling cycle cooler has shown recovery of fungi that was $97 \%$ effective, fungi from all major groups that were recovered, including recognised cryopreservation-recalcitrant strains (10). The control of cooling is much more finite with the Stirling cooler, reducing the variation in ice nucleation between replicates (10).

In this manuscript, we describe cryopreservation with a controlled rate cooler and estimate the genomic content of a selected soil sample before and after cryopreservation. Two methods of cryopreservation were used and compared with control aliquots of soil. Metabarcoding was used to estimate the genomic content of a soil microbiome. We selected a representative soil sample from the Rothamsted collection and used it to estimate the genomic integrity after cryopreservation. The first hypothesis was to confirm the genomic integrity of the microbiome. We also treated the soil sample with a liquid medium to estimate the growth of bacteria and compared their growth before and after cryopreservation. We used PCR-based rRNA metabarcoding to demonstrate that the controlled rate cooler maintains the DNA content of the microbiome.

\section{Methods}

Soil type. Bulk soil samples were collected from a permanently maintained Bare-Fallow (Stackyard, Woburn Experimental Farm, Bedfordshire, UK.) in June 2020. GPS and soil classification data can be found in Table 1.

Sample preparation and cryopreservation. Soil was mixed aseptically with a flame and ethanol sterilised spatula to ensure even distribution of microbial communities, before taking $250 \mathrm{mg}$ aliquots for each sample. Soil samples were cryopreserved with two methods: controlled rate

Page 3 of 17 
cooling (CRC, 'rate'), and plunge cooling (PC, 'plunge'). CRC samples were cooled with a Stirling cycle cooler, Via Freeze Duo (VFD 30006, Cytiva, Amersham, UK) under a standard IMI / CABI culture collection cooling profile of $1^{\circ} \mathrm{C}$ min- 1 (between $5^{\circ} \mathrm{C}$ to $-30^{\circ} \mathrm{C}$ ) outside this range the cooling rate was $2^{\circ} \mathrm{C}$ min-1 and were held at $-80^{\circ} \mathrm{C}$. Plunge cooled samples were fully submerged in dewars filled with liquid nitrogen (LN), samples were taken out once bubbling of LN subsided to pre-sample intensities. When each treatment was completed it was immediately stored in LN vapour phase ultra-cold storage $\left(<175^{\circ} \mathrm{C}\right)$ for 72 hours. When required, both soil sample treatments were thawed by rapid warming in a water bath set to $37^{\circ} \mathrm{C}$ for 5 minutes. Samples were annotated based on their cryopreservation method. Control: no cryopreservation; Plunge: rapidly cooled via PC in LN; Rate: CRC with Stirling cycle cooler (VFD30006).

Enrichment method. Methodology for enrichment was adapted from Yang 2015 (11), $250 \mathrm{mg}$ of soil was added to a falcon tube containing $2.5 \mathrm{ml}$ of 1:10 trypticase soy broth (TSB; Oxoid; $17.0 \mathrm{~g}$ pancreatic digest of casein, 3.0 soya bean, $5.0 \mathrm{~g} \mathrm{NaCl}, 2.5 \mathrm{~g}$ dipotassium hydrogen phosphate, $2.5 \mathrm{~g}$ glucose). This solution was vortexed for 30 seconds, sealed with tape and incubated at $30^{\circ} \mathrm{C}$ at 150 rpm. for 70 hours. Enriched solutions were vacuum filtered using Nalgene ${ }^{\mathrm{TM}}$ Sterile Analytical Filter Units (Thermo Fisher Scientific). Filter paper was used for downstream DNA extractions.

DNA extraction, $P C R$, and NGS library preparation. DNA was isolated from aliquots of $250 \mathrm{mg}$ of the soil sample with DNeasy PowerSoilKit (Qiagen, Manchester, UK) according to the manufacturers' instruction. For the enrichment, a preliminary filtration of the liquid medium was undertaken with nylon Whatman membrane filters with pore size of $0.2 \mu \mathrm{m}$ (Merck Life Science UK Ltd, Gillingham, UK). DNA was quantified using Qubit 3.0 Fluorometer (Thermo Fisher Scientific) and normalised to 5 $\mathrm{ng} / \mu \mathrm{l}$ before PCR reactions. Genomic DNA was amplified with two primer pairs for $16 \mathrm{~S}$ and ITS rRNA genes, for bacterial and fungal amplicons respectively, with primers modified with the Illumina adapter overhang sequences to the $5^{\prime}$-end. Metabarcoding was performed on the V3-V4 16S rRNA region for bacteria with V3F ( $5^{\prime}$-CCTACGGGNGGCWGCAG-3') and V3R (5'GACTACHVGGGTATCTAATCC-3') (12). For the ITS rRNA, the primer were: ITS1 FI2 (5'GAACCWGCGGARGGATCA-3') (13) and ITS2 (5'-GCTGCGTTCTTCATCGATGC-3') (14) (15). PCR was undertaken in a Bioer TC1300 LifeECO Thermal cycler (Alpha Laboratories, Eastleigh, UK) with a reaction mix containing $5 \mu \mathrm{l}$ of each primer at the concentration of $1 \mu \mathrm{M}, 2.5 \mu \mathrm{l}$ of template DNA at the concentration of $5 \mathrm{ng} / \mu \mathrm{l}$, and $12.5 \mu \mathrm{l}$ of KAPA HiFi HotStart ReadyMix (Roche Life Sciences, Welwyn, UK) to a final volume of $25 \mu \mathrm{l}$ with PCR grade water. PCR reactions were preincubated for 3 min at $95^{\circ} \mathrm{C}$ followed by $25 \mathrm{cycles}$ of $30 \mathrm{sec}$ at $95^{\circ} \mathrm{C}, 30 \mathrm{sec}$ at $55^{\circ} \mathrm{C}$, and $30 \mathrm{sec}$ at $72^{\circ} \mathrm{C}$. Samples were finally incubated for $5 \mathrm{~min}$ at $72^{\circ} \mathrm{C}$, followed by chilling to $10^{\circ} \mathrm{C}$. Aliquots of $1 \mu \mathrm{l}$ of index PCR products were assessed for quality with an Agilent Tapestation 4200 (Agilent Technologies), and quantified with Qubit ${ }^{\mathrm{TM}}$ (Thermo Fisher Scientific). Libraries were prepared with a Nextera Flex DNA Library prep kit (Illumina, Cambridge, UK), according to the manufacturers' instruction. Steps included the introduction of indices with amplification of DNA and clean up, normalisation and pool. All libraries were validated with an Agilent Tapestation 4200 (Agilent Technologies Ltd, Stockport, UK) and final Concentration in $\mathrm{nM}$ was calculated based on the size of the library as determined by Agilent Tapestation 4200. Concentrated libraries were diluted to $4 \mathrm{nM}$ with $10 \mathrm{mM}$ Tris pH 8.5. Pooled DNA libraries were combined with $5 \mu \mathrm{l}$ of $0.2 \mathrm{~N} \mathrm{NaOH}$, and incubated for 5 minutes at room temperature to denature the DNA into single strands. Denatured DNA was then diluted to a final 
concentration of $15 \mathrm{pM}$ with Illumina Hybridization buffer (Illumina) and sequenced with the Illlumina MiSeq at CABI (Egham, UK) on an Illumina MiSeq V3 Cartridge (600 cycles) (Illumina).

Metabarcoding data analysis. Using DADA2 v1.16.0 $(16,17)$, sequences were truncated and denoised based on quality score using default values; maximum expected errors for forward and reverse reads were 4 and 7 respectively and no truncation length was set for both ITS and $16 \mathrm{~S}$ sequences. Chimera removal and merge of reads were done with default parameters. $16 \mathrm{~S}$ taxonomy was assigned on representative ASVs against SILVA ref NR dataset v.138 (18). ITS taxonomy was assigned on representative ASVs against UNITE fungal taxonomic reference v8.2 (19). Normalisation of reads, richness and abundance analyses were undertaken with Phyloseq package v1.34.0 (20) and visualised with ggplot2 (v3.3.3). Various helper functions were used for layout of ggplot2 objects with cowplot, manipulating Phyloseq objects to work with other pipelines and calculating error bars.

Statistical analyses. All statistical analyses were performed within the computing environment $R$ ( $v$ 3.5.0; R Core Development Team, 2005) and visualized using ggplot2 (v3.3.3). To visualize the overall bacterial communities, normalised sequence counts from phyloseq were used to plot non-metric multidimensional scaling (NMDS) on Bray-Curtis dissimilarity matrices to ordinate in two dimensions the variance of beta diversity using the Vegan package (v2.5-7). NMDS analysis was performed between samples grouped by cryopreservation treatments on ITS metabarcodes, and between enrichment and cryopreservation treatments on $16 \mathrm{~S}$ metabarcodes. These dissimilarity matrices were also used to analyse the group effects between treatments and enrichment with pairwise permutational analysis of variance (PERMANOVA) using the adnois() function in Vegan and convenience wrapper functions in the pairwise.adonis package (v0.01). Pairwise PERMANOVA analysis was performed between samples grouped by cryopreservation treatments on ITS metabarcodes, and between enrichment and cryopreservation treatments on $16 \mathrm{~S}$ metabarcodes with 999 permutations. P values were false discovery rate corrected by the Benjamini-Hockberg method (21). Heat tree plots were made using phyloseq objects converted to work with metacoder (v0.3.4) (22).

\section{Results}

The impact of cryopreservation on the microbial communities was assessed by comparing the microbiomes using culture-free approaches. Metabarcodes were generated for bacterial (16S rRNA) and fungal (ITS rRNA) amplicons following different treatments of controlled rate cooling (CRC, 'rate'), and plunge cooling (PC, 'plunge'), compared to a non-treatment ambient control (control).

Impact of cooling treatments on microbiome sequence content. A total of 9,846,613 read counts were obtained for $16 \mathrm{~S}$ rRNA, and 8,493,298 for ITS rRNA (Table 2). In total, 4,915,778 sequences were obtained for the 'control', 7,191,917 sequences for the 'plunge' and 6,232,216 sequences for the 'rate' treatments across 44 replicates: five replicates for each treatment and enrichment pair. One replicate of the 'control' was excluded from the data analysis due to sequence data corruption. 


\section{Discussion} produce amplicons.

Enrichment produced PCR amplicon for the 165 rRNA PCR only with ITS rRNA PCR that did not

Richness of the cryopreserved soil microbiome. The overall richness of bacterial ASVs was statistically comparable across the samples $(\mathrm{F}=0.982, \mathrm{p}=0.565)$. Similarly, the richness of eukaryotic ASVs did not produce significant differences ( $F=1.392, p=0.163)$. After enrichment, significant differences were observed $(F=1.851, p=0.014)$. Further analyses were undertaken to compare the treatments with pairwise comparison of the relative abundance of ASVs, which was used to estimate the similarity of the soil microbiome before and after cryopreservation (Table 3). The three treatments 'control, 'plunge' and 'rate' were statistically comparable. After enrichment, there were significant differences in the composition of ASVs. When comparing the datasets after the enrichment step, only 'rate' was statistically comparable to the control $(p=0.219)$. Additional multivariate statistics with non-metric multidimensional scaling supported the similarities of 'plunge' and 'rate' with the 'control' (Figure 1). With the ITS metabarcoding, 'plunge' and 'rate' had similar ASVs composition compared to the 'control' ( $p=0.358$ ) (Table 4).

Taxonomic affiliation of the metabarcodes before and after cryopreservation. When individual ASVs were grouped by taxonomic affiliation, the similarity across the three treatments was supported further (Figure 2). The ten most abundant taxa recovered from the three treatments were, on average, $41.5 \%$ of the total number of DNA reads generated by $16 \mathrm{~S}$ rRNA metabarcoding, and $58.3 \%$ of ITS rRNA. In the $16 \mathrm{~S}$ rRNA metabarcoding, several had little variability across the three treatments: for example, Acidobacteriales were $5.79 \% \pm 0.87$ in the 'control', $5.61 \% \pm 0.43$ in 'plunge' and 5.56\% \pm 0.32 in 'rate'. Tepidisphaerales had more variation and were more abundant in 'rate' (9.88\% \pm 0.26 vs $7.96 \% \pm 1.04$ in 'control' and $7.69 \% \pm 0.90$ in 'plunge') (Figure 2 ). In the ITS rRNA metabarcoding, the ten most abundant genera were consistent in the three treatments (Figure 2). Relative abundances of the ten most abundant genera had minor variations and were consistent overall. For example, the two most abundant genera in the three treatments were Metarhizium sp. and Mortierella sp. Other less abundant genera were consistent in the three treatments.

Microbial growth before and after cryopreservation. With the enrichment method, some soil bacterial taxa led to different trends when comparing 'control' vs 'plunge' and 'control' vs 'rate'. After incubation of soil aliquots in enrichment broth, the ten most abundant taxa recovered from the three treatments, which covered, on average, 93.1\% of the total number of DNA reads, were analysed further. When comparing 'control' vs 'plunge', the biggest variability was seen for Chryseobacterium sp. (-13.9\% after 'plunge') and Enterobacterales ( $+10.2 \%$ after 'plunge'). When comparing 'control' vs 'rate', the variability was smaller than 'plunge' with Chryseobacterium sp. being the most affected genus (-8.4\% after 'rate') and Bacillales ( $+7.6 \%$ after 'rate') (Figure 2$)$. Enterobacterales after 'rate' were similar to those recovered from the 'control' $(-0.3 \%)$. Overall, 'rate' was more similar to the 'control' than 'plunge'. 
We applied an optimised method for cryopreservation for which the genomic integrity of the microbiome was conserved when compared with a 'control' treatment. The optimised cryopreservation of soil samples is essential for the development of microbiome research in order to retain stable, functionally intact microbiomes (7). Our results showed that metabarcoding of $16 \mathrm{~S}$ and ITS rRNA were useful methods to estimate successful cryopreservation. In addition, the microbiome after enrichment delivered a similar response of cryopreserved soil and this was estimated with the comparison of ten most abundant bacterial taxa accounting for, on average, $93.1 \%$ of DNA reads. These findings support a successful process of cryopreservation and are promising for future use of this technology. Previous success on cryopreservation and resuscitation of natural aquatic prokaryotic communities has shown promising results also (2). To the best of our knowledge, this study is the first report of cryopreservation of soil using a Stirling cycle cooling approach. Further, we were able to cryopreserve and resuscitate a soil sample which maintained a representative component of its genomic diversity.

Eukaryotes organisms had a similar response to that of the bacteria. 'Plunge' and 'rate' treatments produced a consistent profile compared to the 'control', showing that these methods maintain the genomic integrity of the eukaryotic portion of the microbiome similarly to the bacteria. This was particularly evident with the comparison of the richness (Figure 1) and confirms previous findings for which 'rate' is the optimal preservation approach for fungi (10). However, the method utilised has yet to be optimised and more tests are required to improve the process. For example, the main aim is to reduce ice formation which can be damaging for the cells in the samples. But mitigating the damaging ice-effect will cause less damage to the organisms within the sample. Recent research by McClure et al. (23), suggests that, under stress, microbial communities within soil samples may produce trehalose, which has been well-characterised as a synthesised osmoprotectant in bacteria (24). This is of significance as trehalose is a natural cryoprotectant and the presence of this would reduce ice damage. Drying of samples to reduce residual moisture content in a controlled way that promotes trehalose formation would therefore be worthy of further investigation.

Intact genomic content was recovered after cryopreservation. 16S rRNA metabarcodes were similar in 'control', 'plunge' and 'rate' with 'plunge' being more similar to the 'control' than 'rate' without enrichment. This trend could be explained by the 'plunge' method which rapidly stops metabolism while 'rate' reaches similar output but gradually. Similarly, ITS rRNA metabarcodes were consistent in 'control', 'plunge' and 'rate'. The overwhelming majority of microbes are essential for ecosystem functioning and known for their interactions with other microorganisms as well as macroorganisms (9), which supports the importance of maintaining the characteristics of a system so that species interactions and communication remain untouched to provide sustainable population dynamics and functional activities (25).

266 For the second hypothesis we compared bacterial growth after enrichment and forced the microbiome in favour of bacterial development. The enrichment method was used as a measure of the success of preservation to estimate bacterial growth before and after cryopreservation, and measure live, metabolically active bacteria from the total gDNA component. This enrichment method is non-selective; it is a rich medium that favours growth of fast-growing bacteria such as the Enterobacterales. Under these conditions, 'plunge' and 'rate' had a different response. Overall, similar microbial growth was obtained before and after cryopreservation, but 'rate' was similar to the 'control' $(p=0.219)$ as compared to 'plunge' $(p=0.044)$. Our data indicated that cryopreservation 
did not significantly impact community composition after the resuscitation process, and this was particularly true for 'rate'. Specifically, Chryseobacterium sp. and Bacillales determined a better recovery for 'rate' compared to 'plunge' (Figure 2). Aquatic microbiomes have shown similar trends with no significant changes found after cryopreservation (2). However, these data reported on genomic DNA isolated after microfiltration and do not provide indication of the preservation of environmental factors and physical microstructures within the microbiome. More tests should be undertaken to investigate the response of the microbiome after cryopreservation and explore the resuscitation of organisms. The condition for the development of more complex eukaryotic organisms remain more challenging.

With this experiment we did not prove that cryopreservation has kept physical space untouched. We proved that the microbiome, under the same pressure generated by the enrichment with a liquid medium, had an identical response and that 'rate' generated a bacterial profile statically comparable to the 'control'. This proved that the microbiome of the selected soil sample reacted similarly to the control, and that the bacterial fraction responded to the stress was statistically the same. The improved integrity for bacteria suggests that the controlled rate cooling approach may greatly improve the stringency of the cryopreservation preservation for prokaryotes. Although with only a limited set of samples, the results provided a benchmark for further optimisation of process, but importantly shows that cryopreservation of soil samples at ultra-low temperature is preferential for conservation of key microbiome resources. This research provides a baseline for the further optimisation of protocols, the development of standards and a long-term strategy for the conservation of agricultural biodiversity.

In conclusion, this study provides evidence of a feasible method for the cryopreservation encompassing controlled rate cooling techniques and resuscitation of a selected exemplar soil microbiome. Similarly to other findings (2), the cryopreservation method developed and applied in this study can be applied to other microbiomes. In particular, the enrichment method could be particularly useful to investigate resuscitation of microbes, with further development that could include different types of pressure beyond selective growth conditions as shown in this manuscript with the enrichment method.

\section{Author statements}

\section{Authors and contributors}

GC designed and performed the experiment, analysed the data, and wrote first draft; JMB performed the experiment, analysed the data; MR designed the experiment; IC provided soil type information; all authors contributed to the final version of the manuscript.

\section{Conflicts of interest}

The authors declare that there are no conflicts of interest

Page 8 of 17 
312 This work was undertaken through the Crop Microbiome Cryobank Project (Reference: BB/T019700/1) supported by the BBSRC under the Bioinformatics and Biological Resources Fund. $C A B I$ is an international intergovernmental organisation, and we gratefully acknowledge the core financial support from our member countries (and lead agencies) including the United Kingdom (Foreign, Commonwealth and Development Office), China (Chinese Ministry of Agriculture), Australia (Australian Centre for International Agricultural Research), Canada (Agriculture and Agri-Food Canada), Netherlands (Directorate-General for International Cooperation) and Switzerland (Swiss Agency for Development and Cooperation). See https://www.cabi.org/about-cabi/who-we-workwith/key-donors/ for full details.

\section{Acknowledgements}

We thank Helen Stewart for technical support.

\section{References}

1. Faust K, Lahti L, Gonze D, de Vos WM, Raes J. Metagenomics meets time series analysis: Unraveling microbial community dynamics. Curr Opin Microbiol [Internet]. 2015;25(May):5666. Available from: http://dx.doi.org/10.1016/j.mib.2015.04.004

2. Rain-Franco A, de Moraes GP, Beier S. Cryopreservation and Resuscitation of Natural Aquatic Prokaryotic Communities. Front Microbiol. 2021;11(January):1-19.

3. Busby PE, Soman C, Wagner MR, Friesen ML, Kremer J, Bennett A, et al. Research priorities for harnessing plant microbiomes in sustainable agriculture. PLoS Biol. 2017;15(3):1-14.

4. Berg G, Rybakova D, Grube M, Köberl M, Price A. Europe PMC Funders Group The plant microbiome explored : implications for experimental botany. 2017;67(4):995-1002.

5. Cavicchioli R, Ripple WJ, Timmis KN, Azam F, Bakken LR, Baylis M, et al. Scientists' warning to humanity: microorganisms and climate change. Nat Rev Microbiol [Internet]. 2019;17(9):56986. Available from: http://dx.doi.org/10.1038/s41579-019-0222-5

6. Prosser JI, Bohannan BJM, Curtis TP, Ellis RJ, Firestone MK, Freckleton RP, et al. Prosser et al 2007_The role of ecological theory in microbial ecology. Nat Rev Microbiol. 2007;5(May):384-92.

7. Ryan MJ, Schloter M, Berg G, Kostic T, Kinkel LL, Eversole K, et al. Development of Microbiome Biobanks - Challenges and Opportunities. Trends Microbiol [Internet]. 2021;29(2):89-92. Available from: https://doi.org/10.1016/j.tim.2020.06.009 
8. Gich F, Janys MA, König M, Overmann J. Enrichment of previously uncultured bacteria from natural complex communities by adhesion to solid surfaces. Environ Microbiol [Internet]. 2012 Nov 1;14(11):2984-97. Available from: https://doi.org/10.1111/j.14622920.2012.02868.x

9. Berg G, Rybakova D, Fischer D, Cernava T, Vergès MCC, Charles $T$, et al. Microbiome definition re-visited: old concepts and new challenges. Microbiome. 2020;8(1):1-22.

10. Ryan MJ, Kasulyte-Creasey D, Kermode A, San SP, Buddie AG. Controlled rate cooling of fungi using a stirling cycle freezer. Cryo-Letters. 2014;35(1):63-9.

11. Yang F, Liu HM, Zhang R, Chen D Bin, Wang X, Li SP, et al. Chryseobacterium shandongense sp. nov., isolated from soil. Int J Syst Evol Microbiol. 2015;65(6):1860-5.

12. Klindworth A, Pruesse E, Schweer T, Peplies J, Quast C, Horn M, et al. Evaluation of general $16 \mathrm{~S}$ ribosomal RNA gene PCR primers for classical and next-generation sequencing-based diversity studies. Nucleic Acids Res. 2013;41(1):1-11.

13. Schmidt P-A, Bálint M, Greshake B, Bandow C, Römbke J, Schmitt I. Illumina metabarcoding of a soil fungal community. Soil Biol Biochem [Internet]. 2013;65:128-32. Available from: https://www.sciencedirect.com/science/article/pii/S0038071713001867

14. White T, Bruns T, Lee $S$, Taylor J. Amplification and direct sequencing of fungal ribosomal RNA genes for phylogenetics. New York, USA: Academic Press; 1990. 315-322 p.

15. Cafà G, Caggiano B, Reeve MA, Bhatti H, Honey SF, Bajwa B, et al. A polyphasic approach aids early detection of potentially toxigenic aspergilli in soil. Microorganisms. 2019;7(9).

16. Callahan BJ, McMurdie PJ, Rosen MJ, Han AW, Johnson AJA, Holmes SP. DADA2: Highresolution sample inference from Illumina amplicon data. Nat Methods [Internet]. 2016;13(7):581-3. Available from: https://doi.org/10.1038/nmeth.3869

17. Prodan A, Tremaroli V, Brolin H, Zwinderman AH, Nieuwdorp M, Levin E. Comparing bioinformatic pipelines for microbial $16 S$ rRNA amplicon sequencing. PLoS One. 2020;15(1):119.

18. Quast C, Pruesse E, Yilmaz P, Gerken J, Schweer T, Yarza P, et al. The SILVA ribosomal RNA gene database project: Improved data processing and web-based tools. Nucleic Acids Res. 2013;41(D1):590-6.

19. Abarenkov K, Henrik Nilsson R, Larsson K-H, Alexander IJ, Eberhardt U, Erland S, et al. The UNITE database for molecular identification of fungi - recent updates and future perspectives. New Phytol [Internet]. 2010 Apr 1;186(2):281-5. Available from: https://doi.org/10.1111/j.1469-8137.2009.03160.x

20. McMurdie PJ, Holmes S. Phyloseq: An R Package for Reproducible Interactive Analysis and Graphics of Microbiome Census Data. PLoS One. 2013;8(4).

21. Benjamini Y, Hochberg Y. Controlling the False Discovery Rate: A Practical and Powerful Approach to Multiple Testing. J R Stat Soc Ser B [Internet]. 1995 May 4;57(1):289-300. 
22. Foster ZSL, Sharpton TJ, Grünwald NJ. Metacoder: An R package for visualization and manipulation of community taxonomic diversity data. PLoS Comput Biol. 2017;13(2):1-15.

23. McClure RS, Lee JY, Chowdhury TR, Bottos EM, White RA, Kim YM, et al. Integrated network modeling approach defines key metabolic responses of soil microbiomes to perturbations. Sci Rep [Internet]. 2020;10(1):1-9. Available from: https://doi.org/10.1038/s41598-020-67878-7

24. Giaever HM, Styrvold OB, Kaasen I, Strøm AR. Biochemical and genetic characterization of osmoregulatory trehalose synthesis in Escherichia coli. J Bacteriol. 1988;170(6):2841-9.

25. Bassler BL. Small talk: Cell-to-cell communication in bacteria. Cell. 2002;109(4):421-4. 


\section{$394 \quad$ Figures and tables}

395

396

Table 1. Soil sample used in this study: site Stackyard, at the time of sampling land use was

397 "permanent Bare-Fallow", Woburn Experimental Farm, Stackyard field, Bedfordshire, UK.

\begin{tabular}{|l|l|l|l|l|l|l|l|l|}
\hline \multirow{2}{*}{$\begin{array}{l}\text { Collection } \\
\text { Date }\end{array}$} & \multirow{2}{*}{$\begin{array}{l}\text { Soil_taxonomic } \\
\text { classification }\end{array}$} & \multicolumn{4}{|l|}{ Geographic location } & \multicolumn{2}{|l|}{ Texture } \\
\cline { 3 - 9 } $16 / 06 / 2020$ & Depth & Elevation & Latitude & Longitude & Sand & Silt & Clay \\
\hline & $\begin{array}{l}\text { Sandy loam } \\
\text { series) }\end{array}$ & $0-20 \mathrm{~cm}$ & $100 \mathrm{~m}$ & 52.0004 & 0.614265 & $50 \%$ & $27 \%$ & $18 \%$ \\
\hline
\end{tabular}

400

401 
402

403

404 Table 2. Raw statistics of metabarcodes obtained in this study

\begin{tabular}{|c|c|c|c|c|}
\hline Treatment & Replicate & $16 S$ & 16S_enrich & ITS \\
\hline \multirow[t]{5}{*}{ Control } & 1 & 246,069 & 267,068 & 127,049 \\
\hline & 2 & 160,161 & 416,846 & 263,067 \\
\hline & 3 & 435,272 & 576,261 & 77,441 \\
\hline & 4 & - & 520,926 & 719,407 \\
\hline & 5 & 194,165 & 301,919 & 610,127 \\
\hline \multirow[t]{5}{*}{ Plunge } & 1 & 183,771 & 350,703 & $1,755,679$ \\
\hline & 2 & 446,374 & 163,302 & 447,575 \\
\hline & 3 & 396,369 & 360,018 & 270,863 \\
\hline & 4 & 200,425 & 386,624 & 604,740 \\
\hline & 5 & 625,850 & 566,661 & 432,963 \\
\hline \multirow[t]{5}{*}{ Rate } & 1 & 195,671 & 144,839 & $1,186,559$ \\
\hline & 2 & 260,284 & 333,558 & 389,127 \\
\hline & 3 & 179,039 & 322,090 & 788,068 \\
\hline & 4 & 578,534 & 398,167 & 453,732 \\
\hline & 5 & 264,684 & 370,963 & 366,901 \\
\hline
\end{tabular}

405

406

Page 13 of 17 
407

408

409

410

411

412

\begin{tabular}{|c|c|c|c|c|c|c|}
\hline & $C(16 S)$ & $P(16 S)$ & $R(16 S)$ & CE(16S) & $\mathrm{PE}(16 \mathrm{~S})$ & $\operatorname{RE}(16 S)$ \\
\hline$C(16 S)$ & - & - & - & - & - & - \\
\hline$P(16 S)$ & 0.632 & - & - & - & - & - \\
\hline$R(16 S)$ & 0.371 & 0.701 & - & - & - & - \\
\hline CE(16S) & 0.017 & 0.018 & 0.017 & - & - & - \\
\hline PE(16S) & 0.017 & 0.017 & 0.017 & 0.044 & - & - \\
\hline $\operatorname{RE}(16 \mathrm{~S})$ & 0.017 & 0.017 & 0.017 & 0.219 & 0.043 & - \\
\hline
\end{tabular}

413

414 
416 Table 4. Pairwise comparisons of corrected $P$ values by PERMANOVA analysis based on Bray-Curtis 417 dissimilarity matrix of normalised ITS sequence counts. P Value correction for false discovery rate 418 preformed with Benjamini-Hochberg correction. C: No cryopreservation ('control'); P: Plunge cooled 419 in LN ('plunge'); and R: Controlled rate cooled ('rate').

420

\begin{tabular}{l|ccc}
\hline & \multicolumn{1}{|c}{ (ITS) } & $\mathbf{P ( I T S )}$ & $\mathbf{R}($ ITS) \\
\hline $\mathbf{C}($ ITS $)$ & - & - & - \\
$\mathbf{P}($ ITS $)$ & 0.358 & - & - \\
$\mathbf{R}($ ITS $)$ & 0.358 & 0.358 & - \\
\hline
\end{tabular}

421

422 

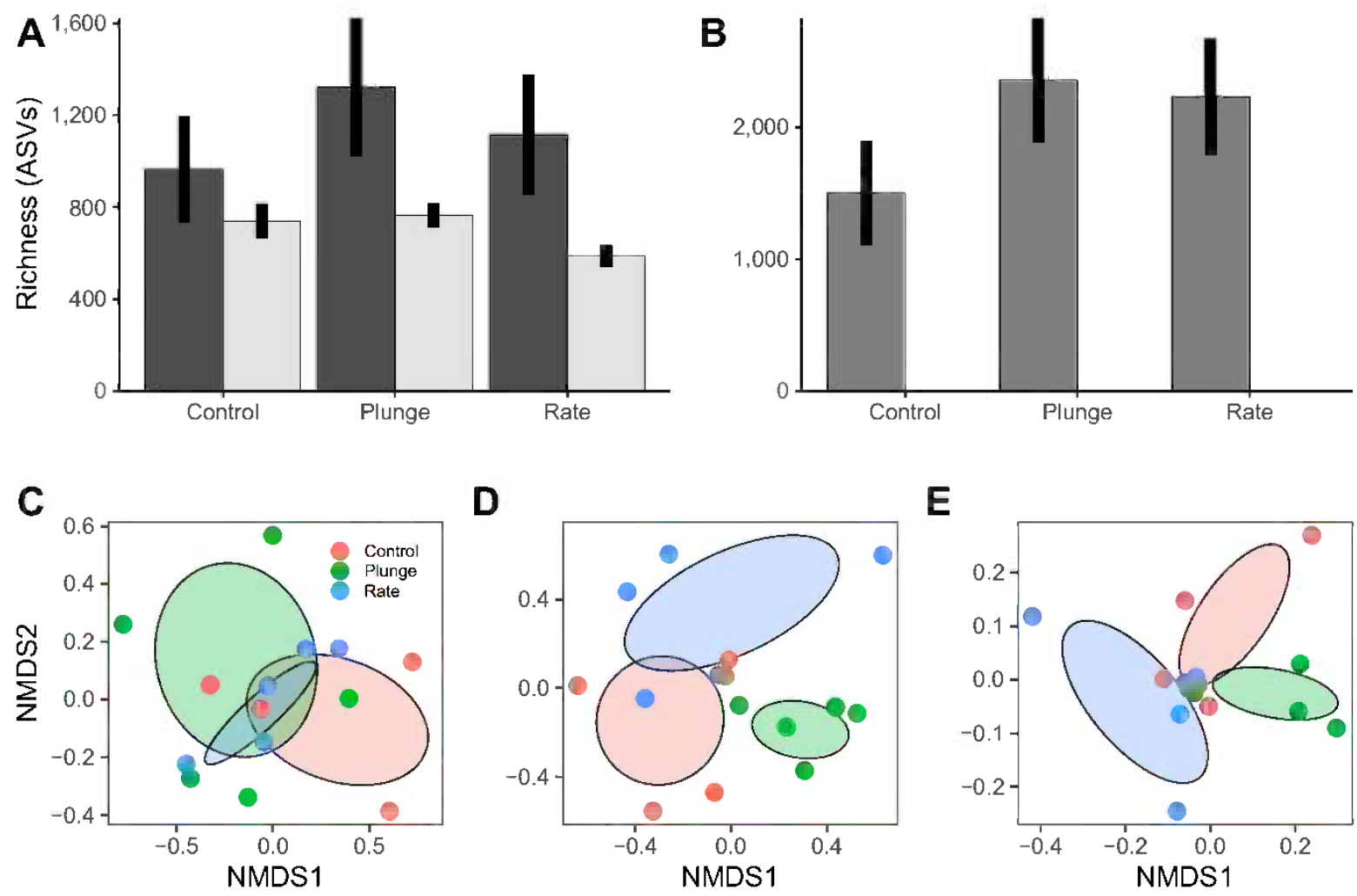

Figure 1. Richness of bacteria (A) and eukaryotes (B) of soil across different cryopreservation treatments. Control: No cryopreservation; Plunge: Plunged cooled in LN; Rate: Controlled rate cooled. Richness of non-enriched (dark bars) and enriched soil (light bars). Error bars are bootstrapped 95\% confidence intervals implemented in ggplot2; C-E) Non-metric multidimensional scaling (NMDS) of 16S (C), Enriched 16S (D) and ITS (E) rRNA metabarcodes of soil across different cryopreservation treatments. NMDS was derived from Bray-Curtis dissimilarity matrices calculated from ASV sequence proportions among samples. Points are sample replicates and coloured ellipses are calculated with standard deviation of sample centroids for each cryopreservation treatment using 'ordiellipses' in the vegan package. 


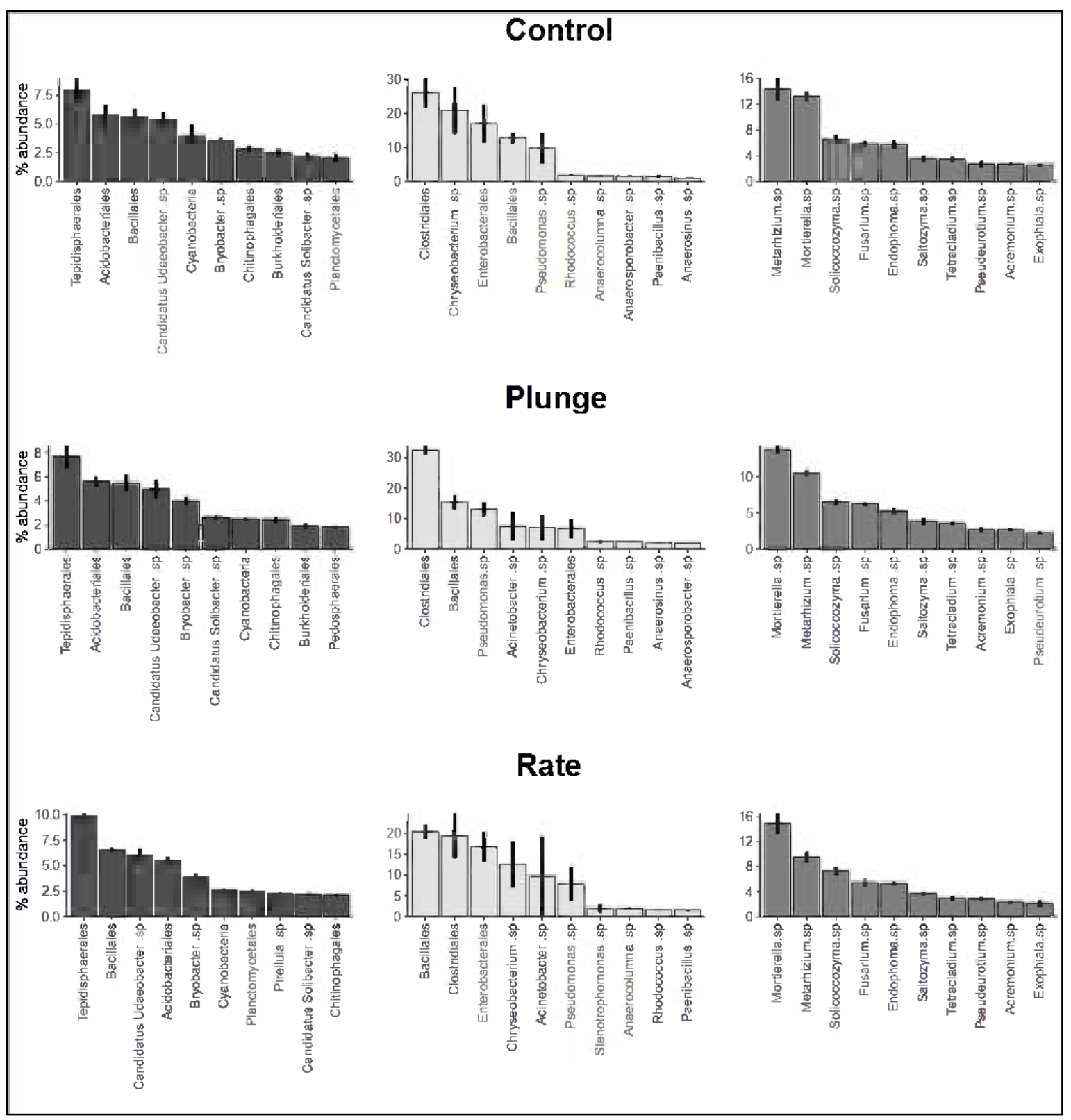

Figure 2. Relative abundance of dominant bacterial ASVs in control soil (left panels), enriched soil (central panels) and eukaryotic (right panels) across different cryopreservation treatments. Error bars are bootstrapped $95 \%$ confidence intervals implemented in ggplot2. 